\title{
A SPECTROPOLARIMETRIC VIEW ON THE NATURE OF THE PECULIAR TYPE I SN 2005hk*
}

\author{
Justyn R. Maund ${ }^{1,2,8}$, J. Craig Wheeler $^{3}$, Lifan Wang ${ }^{4}$, Dietrich BaAde ${ }^{5}$, Alejandro Clocchiatti ${ }^{6}$, \\ Ferdinando Patat ${ }^{5}$, Peter Höflich ${ }^{7}$, Jason QuinN $^{6}$, and Paula Zelaya ${ }^{6}$ \\ ${ }^{1}$ Dark Cosmology Centre, Niels Bohr Institute, University of Copenhagen, Juliane Maries Vej, DK-2100 Copenhagen Ø, Denmark; justyn@dark-cosmology.dk \\ 2 Department of Astronomy \& Astrophysics, University of California, Santa Cruz, CA 95064, USA \\ ${ }^{3}$ Department of Astronomy and McDonald Observatory, The University of Texas, 1 University Station C1402, Austin, TX 78712-0259, USA; \\ wheel@astro.as.utexas.edu \\ ${ }^{4}$ Department of Physics, Texas A\&M University, College Station, TX 77843-4242, USA; wang@ physics.tamu.edu \\ ${ }^{5}$ ESO-European Organization for Astronomical Research in the Southern Hemisphere, Karl-Schwarzschild-Str. 2, 85748 Garching b. München, Germany; \\ dbaade@eso.org,fpatat@eso.org \\ ${ }^{6}$ Departamento de Astronoma y Astrofisica, PUC Casilla 306, Santiago 22, Chile; aclocchi@astro.puc.cl, jquinn@astro.puc.cl, pazelaya@ astro.puc.cl \\ ${ }^{7}$ Department of Physics, Florida State University, Tallahassee, FL 32306-4350, USA; pah@ astro.physics.fsu.edu \\ Received 2010 May 6; accepted 2010 August 21; published 2010 September 27
}

\begin{abstract}
We report two spectropolarimetric observations of SN 2005hk, which is a close copy of the "very peculiar" SN 2002cx, showing low peak luminosity, slow decline, high ionization near peak, and an unusually low expansion velocity of only about $7000 \mathrm{~km} \mathrm{~s}^{-1}$. Further to the data presented by Chornock et al., at -4 days before maximum, we present data of this object taken on 2005 November 9 (near maximum) and November 23 (+2 weeks) that show the continuum and most of the spectral lines to be polarized at levels of about $0.2 \%-0.3 \%$. At both epochs the data correspond to the Spectropolarimetric Type D1. The general low level of line polarization suggests that the line-forming regions for most species observed in the spectrum have a similar shape to that of the photosphere, which deviates from a spherical symmetry by $<10 \%$. In comparison with spectropolarimetry of Type Ia and corecollapse SNe at similar epochs, we find that the properties of SN 2005hk are most similar to those of Type Ia SNe. In particular, we find the low levels of continuum and line polarization to indicate that the explosion mechanism is approximately spherical, with homogeneous ejecta (unlike the chemically segregated ejecta of CCSNe). We discuss the possibility that SN 2005hk was the result of the pure deflagration of a white dwarf and note the issues concerning this interpretation.
\end{abstract}

Key words: supernovae: general

Online-only material: color figures

\section{INTRODUCTION}

The nature of the complex explosion mechanisms thought to be behind supernovae ( $\mathrm{SNe}$ ), of all types, is expected to leave a signature in the resulting geometry of the explosion. The study of the polarized light arising from $\mathrm{SN}$ explosions provides a unique and powerful method for directly measuring asymmetries in $\mathrm{SNe}$. The power of spectropolarimetry is demonstrated by its application to the study of the shapes of SNe at large distances, providing an effective spatial resolution far superior to any current or currently planned imaging technique (see Wang \& Wheeler 2008, for a review).

In $\mathrm{SN}$ photospheres, the predominant form of opacity is due to electron scattering, which is also a polarizing process. In a spherical symmetry, light originating from different quadrants of the photosphere have opposite but equal polarization components, leading to a zero net observed polarization. In the presence of asymmetries, the cancellation of the polarization components arising from different portions of the ejecta is incomplete, leading to an observable polarization whose magnitude is related to the degree of the asymmetry. In addition to the polarization of continuum light, the absorption due to line-forming species exterior to the photosphere casts shadows on the photosphere. If the photosphere is not completely covered, a polarization signature will be associated with wavelength-dependent absorption features, with the degree of polarization related to the amount

\footnotetext{
* Based on observations made with ESO Telescopes at the Paranal Observatory, under program 076.D-0178(A).

8 Sophie \& Tycho Brahe Fellow.
}

and orientation of the coverage of the photosphere by the lineforming region.

Previous polarimetric studies of SNe have shown that Type Ia $\mathrm{SNe}$ and core-collapse supernovae (CCSNe) have different polarization properties. Type Ia SNe are observed to have generally low polarizations, indicative of a close-to-spherical symmetry. The degree of polarization of these events decreases with time, as deeper layers of ejecta are revealed, implying the ionization/ density structure becomes more spherically symmetric with depth. In contrast, CCSNe have been observed with a wide variety of polarizations, generally larger than those observed for Type Ia SNe. In addition, the degree of polarization is observed to increase with time for CCSNe, suggesting the ejecta become more asymmetric at increasing depth.

In the classical observing modes of photometry and spectroscopy, Type Ia SNe at certain phases can be confused upon casual inspection with Type Ic $\mathrm{SNe}$ at other phases due to the common display of $\mathrm{Fe}$ II lines. Special care must be given when trying to determine the type and the physics of "peculiar" Type Ia and "peculiar" Type Ic SNe. The application of spectropolarimetry can provide additional constraints, both in the abstract sense of the Stokes parameters and in the inferred geometries, that can help separate events belonging to the different distinct classifications.

One particular class of $\mathrm{SNe}$, which have been previously classified as Type Ia SNe (albeit "the most peculiar Type Ia;" $\mathrm{Li}$ et al. 2003), are the so-called 2002cx-like SNe. It has recently been suggested that members of this class may be representative of Type Ia SN events that result from a pure deflagration (see 


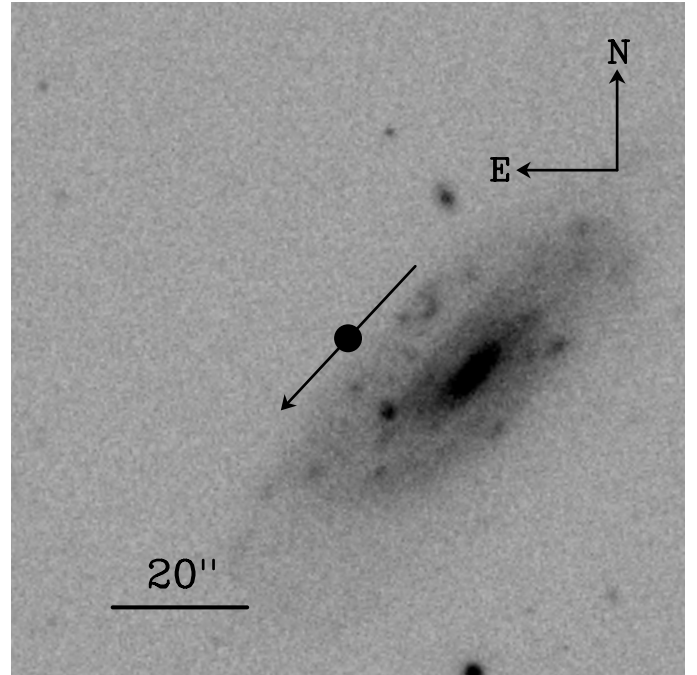

Figure 1. SDSS $r^{\prime}$-band image of the site of SN 2005hk, indicated by the filled circle, relative to its host galaxy UGC 272. The orientation of the determined ISP component, at P.A. $=141 \pm 3^{\circ}$, is shown by the arrow.

Branch et al. 2004, and references therein) or instead may be peculiar, low-luminosity Type Ic SNe (Foley et al. 2009; Valenti et al. 2009).

Here, we report two epochs of spectropolarimetry of the 2002cx-like SN 2005hk, acquired at approximately maximum light and two weeks later using the Very Large Telescope (VLT) of the European Southern Observatory (ESO). SN 2005hk was discovered in UGC 272 by Burket \& Li (2005). The location of SN 2005hk, relative to its host galaxy, is shown in Figure 1. Early spectroscopy was presented by Jha et al. (2006), Chornock et al. (2006), Phillips et al. (2007), and Sahu et al. (2008). They identify the early high ionization (e.g., Fe III) species typical of SN 2002cx-like SNe, as well as the bright Type Ia SN $1991 \mathrm{~T}$ (see also Serduke et al. 2005), and the low velocities characteristic of SN $2002 \mathrm{cx}\left(\sim 6000 \mathrm{~km} \mathrm{~s}^{-1}\right)$. The spectra of Jha et al. and Chornock et al. showed intermediate-mass elements with weaker features than regular $\mathrm{SNe} \mathrm{Ia}$, but stronger than SN 1991T. Chornock et al. suggested that Ni and Co may be present in the early spectra and hence in the outermost layers of the ejecta. The red portions of the spectra show appreciable features of O I and $\mathrm{Mg}$ II between 7500 and $9000 \AA$, whereas SN 1991T, a possible analog, showed a basically featureless spectrum in that phase and wavelength range. Both SN 2002cx and SN 2005hk showed similar fine scale structure suggesting that the structure was systematic and hence not the product of random clumping in the ejecta of either event. Phillips et al. (2007) present extensive $u^{\prime} g^{\prime} r^{\prime} i^{\prime} B V R I J H K$ photometry and optical spectroscopy of SN 2005hk, including the spectral data of Jha et al. and Chornock et al. Importantly, they note that the light curves of these two events are very similar, with neither showing the typical secondary maxima in $R$ and $I$ displayed by normal SNe Ia, but light curves that are also distinctly different than under-luminous SNe Ia such as SN 1991bg. In addition, the bolometric light curve, including Swift UV data, shows a rather slow light curve $\left(\Delta m_{15}(B)=1.56\right.$; Phillips et al. 2007), uncharacteristic of under-luminous SN Ia and similar to normal Type Ia $\mathrm{SNe}$ over a magnitude brighter at maximum.

Chornock et al. (2006) presented spectropolarimetry of SN 2005hk, at four days prior to our first observation. They determined that the continuum was polarized $\sim 0.4 \%$ in the red, with the data represented by a dominant axis. They also
Table 1

Spectropolarimetry of SN $2005 \mathrm{hk}$

\begin{tabular}{lcccc}
\hline \hline Object & $\begin{array}{c}\text { Date } \\
\text { UT }\end{array}$ & $\begin{array}{c}\text { Exposure } \\
(\mathrm{s})\end{array}$ & $\begin{array}{c}\text { Median } \\
\text { Airmass }\end{array}$ & Type \\
\hline SN 2005hk & 2005 Nov 09.163 & $4 \times 900$ & 1.255 & SN \\
HD 49798 & 2005 Nov 09.363 & 5 & 1.067 & Flux Std. \\
SN 2005hk & 2005 Nov 23.096 & $4 \times 900$ & 1.160 & SN \\
LTT 1020 & 2005 Nov 23.132 & 5 & 1.139 & Flux Std. \\
\hline
\end{tabular}

identified a weak modulation of the polarization of the Fe III line at $\lambda 5129$.

In Section 2, we present our spectropolarimetric observations and our adopted reduction procedure. The results from these observations are presented in Section 3 and these results are discussed in Section 4. In Section 5, we present our conclusions.

\section{OBSERVATIONS}

Spectropolarimetry of SN 2005hk was acquired using the ESO-VLT on 2005 November 9 and 23, with the FORS1 instrument in polarization PMOS mode. A log of these observations is presented in Table 1. The data were reduced following the scheme outlined in Maund et al. (2007a). At each epoch, the observations consisted of four exposures with the half-wavelength retarder plate positioned at four position angles (P.A.s) $0^{\circ}, 45^{\circ}$, 22.5 , and 67.5, to derive the two linear Stokes parameters $Q$ and $U .{ }^{9}$ Both observations were conducted with the $300 \mathrm{~V}$ grism, without an order separation filter, providing a spectral resolution of $12.5 \AA$ as measured from arc line calibration data. Telluric lines were removed from flux spectra in the standard manner. Flux standard stars were observed, with the full polarimetry optics in place, to calibrate the flux levels of the SN data and remove telluric features. The telluric features are not expected to affect the polarimetry, except to decrease the level of signalto-noise ratio $(\mathrm{S} / \mathrm{N})$ across the absorption, provided there is no significant change in the strength of absorptions between exposures with the retarder plate rotated by $45^{\circ}$.

The instrumental stability between the observations at the two epochs was assessed by calculating the difference between the instrumental signature corrections $\epsilon_{Q}$ and $\epsilon_{U}$ (Maund 2008). At both epochs the difference was found to be $0 \% \pm 0.1 \%$, implying a limit on the instrumental stability and the measurement of the Stokes parameters to $0.1 \%$. This is consistent with previous reported measurements of the instrumental signature corrections and limits on stability of the VLT FORS instrument (Maund et al. 2009).

The degree of polarization was corrected for biases due to observational noise using the equations derived in Wang et al. (1997), and, additionally, using a Monte Carlo simulation of the FORS1 instruments in the manner of Patat \& Romaniello (2006) and Maund (2008). The wavelength scales were corrected for the heliocentric recessional velocity of the host galaxy of 3895 $\mathrm{km} \mathrm{s}^{-1} \cdot 10$

\section{OBSERVATIONAL RESULTS}

\subsection{The Distinguishing Spectral Features}

The spectroscopic properties of SN 2005hk have already been described in some detail in other works (Chornock et al. 2006;

\footnotetext{
9 We adopt the notation of Wang \& Wheeler (2008), where the normalized Stokes parameters are given by $Q=\widehat{Q} / I$ and $U=\widehat{U} / I$.

10 http://nedwww.ipac.caltech.edu/
} 


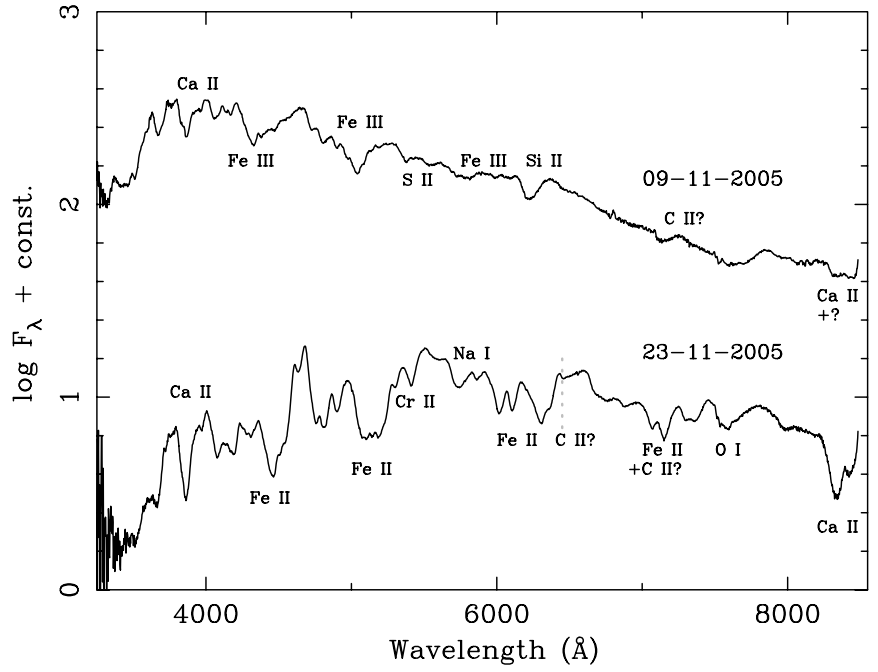

Figure 2. Flux spectra of SN 2005hk at 2005 November 9 and 2005 November 23 , or approximately at $B$ light curve maximum and 14 days after maximum. Line identifications are based on those provided by Chornock et al. (2006), Phillips et al. (2007), and Branch et al. (2004) and fits to the spectra using the SYNOW code. The wavelength scale has been corrected for the recessional velocity of the host galaxy.

Phillips et al. 2007). Here the prominent spectroscopic features, which characterize SN 2005hk at the epochs at which spectropolarimetry was acquired, will be briefly reviewed to orient the reader. The flux spectra from our two spectropolarimetric observations are presented in Figure 2.

Our data from 2005 November 9 are interleaved with the coverage presented by Phillips et al. (2007) who give spectra from November 7 and 14 that they assign to -3 and +4 days with respect to $B$ maximum which they determine to be JD 2,453,685.1. This means our data are from very near $B$ maximum, perhaps a day earlier. We confirm that the spectral features are very similar to those of SN 2002cx at the same epoch (Li et al. 2003). Furthermore, Chornock et al. (2006) acquired a flux spectrum of SN 2005hk in the course of acquiring spectropolarimetry at November 5.4, four days prior to our first observation. Chornock et al. performed line identifications using the SYNOW ${ }^{11}$ code for their spectrum. They found that the strongest lines were from Fe III with other lines of intermediate-mass elements being present, but relatively weaker. They obtained a better fit in the blue by adding lines of Ni II, Co II, Ti II, and C III. They used an excitation temperature of $10,000 \mathrm{~K}$ for the low-ionization stage elements and $15,000 \mathrm{~K}$ for the higher ionization stages, Fe III and Si III. Our flux spectrum from the 2005 November 9 observation, despite being acquired four days later, requires similar identifications to those made by Chornock et al. In the red portion of the spectrum, we identify the Ca II IR triplet, although the synthetic spectra of Chornock et al. suggested that at the epoch of their observations this was mostly due to $\mathrm{O}$ I and $\mathrm{Mg}$ II. The feature at $\sim 7600 \AA$ is identified by Phillips et al. (2007) as Fe II, while Chornock et al. (2006) suggested it was a mixture of O I and $\mathrm{Mg}$ II. Li et al. (2003) observed the appearance of O I $\lambda 7774$ after maximum in spectra of SN 2002cx, which suggests that at 0 days for SN 2005hk this feature could be at least partially due to O I.

Our spectrum from 2005 November 23 corresponds to an epoch two weeks after B maximum. Our data are very similar to spectra of that date presented by Phillips et al. (2007), but our

\footnotetext{
11 http://www.nhn.ou.edu/ parrent/synow.html
}

data have higher $\mathrm{S} / \mathrm{N}$ and broader wavelength coverage. The spectrum of Phillips et al. 2007 from November 25 (+15 days) has similar wavelength coverage to ours of November 23 and remains very similar to our flux spectrum. At this epoch, the underlying continuum is cooler with a relative paucity of flux in the blue compared to the spectrum of November 9. The spectrum is characterized by the growth in amplitude of small-scale features. SYNOW was used by us, in conjunction with previously published line identifications, to determine line identifications specific to the spectrum at this epoch. The degree of blending of multiple lines is lower at the second epoch, due to the relative decrease in the expansion velocity. The truncation of the red side of the O I $\lambda 7774$ emission line profile at $7980 \AA$ may be evidence for $\mathrm{Mg}$ II, but it is located close to strong telluric absorption features. The identification of Si II $\lambda 6355$ at +14 days is debatable and is likely to be due to an increase in Fe II $\lambda \lambda 6456,6518$ (Li et al. 2003). Branch et al. (2004) similarly found that Si II was no longer required to fit spectra of SN 2002cx at a similar epoch. The photospheric velocity was estimated, using lines of Fe II(42), to be $\sim-7000 \mathrm{~km} \mathrm{~s}^{-1}$.

At the second epoch, there are also two distinct absorption features that are likely to be caused by the Ca II IR triplet. The Ca II IR triplet was identified at 12 days after maximum in the spectrum of SN 2002cx (Branch et al. 2004). The two features might be due to partially resolving the triplet in this low velocity photosphere $\left(-5650 \mathrm{~km} \mathrm{~s}^{-1}\right)$, or there could be two kinematically separate components such as those observed in normal Type Ia SNe (Mazzali et al. 2005). If the latter, the absorption minima correspond to velocities of -8400 and $-5500 \mathrm{~km} \mathrm{~s}^{-1}$, respectively, taking the mean wavelength of the Ca II blend to be $8579 \AA$. The bluer of these two components is moving somewhat more rapidly than the typical range identified for photospheric features for Type Ia $\mathrm{SNe}$ at similar epochs (Mazzali et al. 2005). Comparison with SN 2002cx at 12 days (Branch et al. 2004) leads to the identification of the strong absorption observed at $5730 \AA$ as $\mathrm{Na}$ I intrinsic to the SN, corresponding to a velocity of $-8150 \mathrm{~km} \mathrm{~s}^{-1}$ (which is similar to the high-velocity $\mathrm{Ca}$ II feature).

At the first epoch, there is a feature at $\sim 7200 \AA$. Chornock et al. (2006) argue that it is unlikely to be due to $\mathrm{C}_{\text {II }}$ due to the absence of other stronger lines that should occur in the wavelength range of these observations (e.g., $\lambda 6578$ ). Rather Sahu et al. (2008) suggests the $7200 \AA$ may arise from Fe II $\lambda 7308$. Branch et al. (2004) could not identify $\mathrm{C}_{\mathrm{I}}$ features in spectra of SN 2002cx. At the second epoch, however, there is a suggestion of an absorption (blended with an Fe II line giving rise to a flat topped emission line profile for that line) which is consistent with the expected position of $\mathrm{C}_{\text {II }} \lambda 6578$ with a velocity of $-5500 \mathrm{~km} \mathrm{~s}^{-1}$ (similar to the low velocity component of $\mathrm{Ca}$ II).

\subsection{The Polarization of SN $2005 \mathrm{hk}$}

Spectropolarimetry of SN 2005hk at the two epochs is presented in Figures 3 and 4.

The correct measurement of the intrinsic polarization of $\mathrm{SNe}$ requires the subtraction of the polarization contribution of the interstellar medium (ISM), both in the Galaxy and in the host. In the first instance, a general limit can be placed on the degree of the interstellar polarization (ISP) by the amount of reddening measured toward SN 2005hk. For Galactic-type dust the maximum degree of the ISP is related to the reddening by the limit $p_{\text {ISP }}<9 \times E(B-V)$ (Serkowski et al. 1975). The degree of Galactic foreground reddening in the direction of SN $2005 \mathrm{hk}$ 


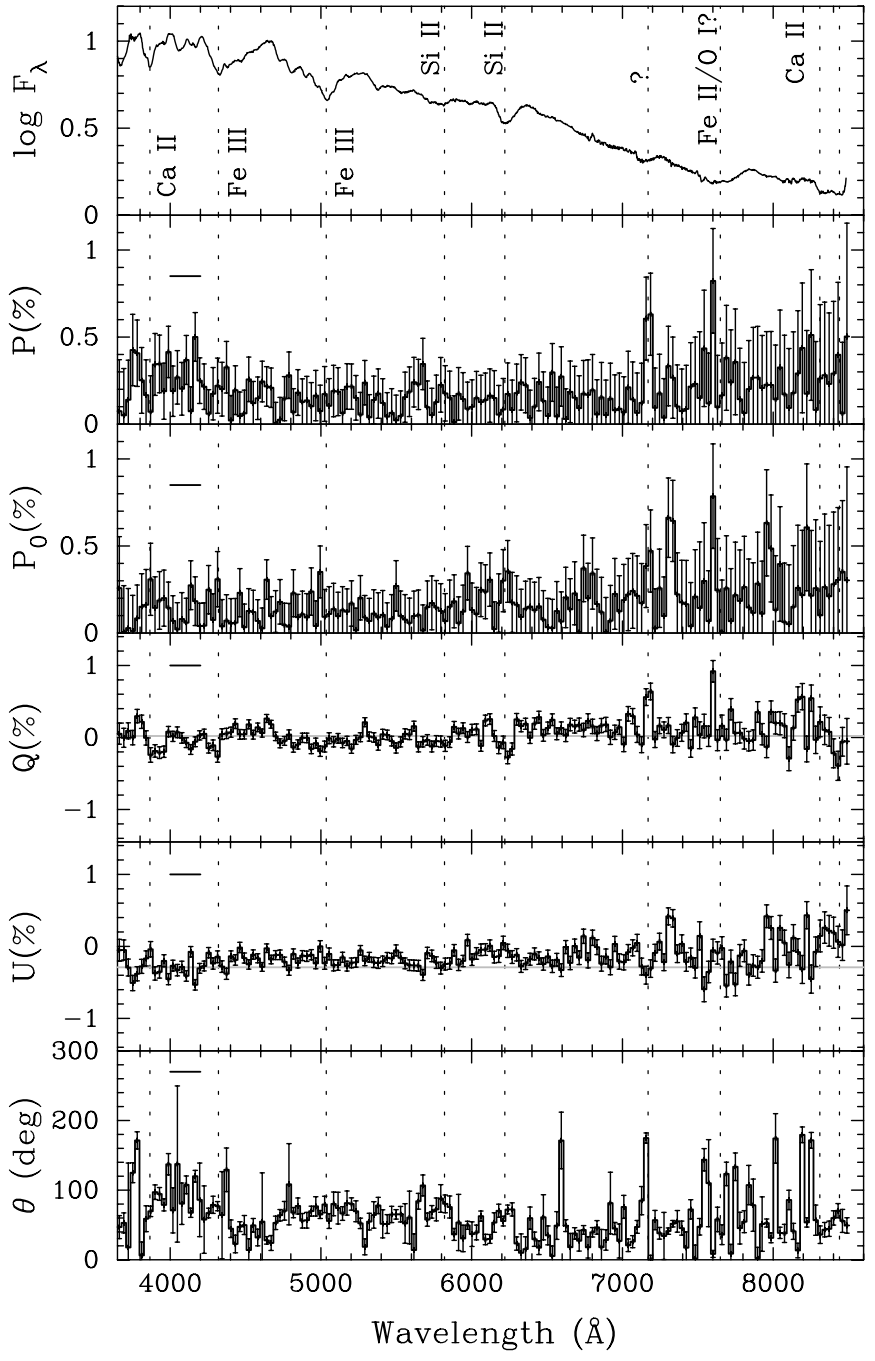

Figure 3. Spectropolarimetry of SN 2005hk on 2005 November 9. The six panels (from top to bottom) give the observed flux spectrum, in logarithmic flux units $\left(\mathrm{erg} \mathrm{s}^{-1} \mathrm{~cm}^{-2} \AA^{-1}\right)$; the observed polarization spectrum $(P)$; the observed polarization spectrum corrected for the ISP component $\left(P_{0}\right)$; the Stokes $Q$ and $U$ spectra uncorrected for the ISP (the value of the ISP is shown for each parameter by the gray line); and the polarization angle $\theta$. Line identifications are provided in the top panel and are based on the identifications by Chornock et al. (2006), Branch et al. (2004), Phillips et al. (2007) and comparison with synthetic spectra produced using the SYNOW code. The data have been rebinned to $30 \AA$ for clarity. The wavelength range selected for the determination of the ISP is shown by the heavy lines.

has been reported to be $E(B-V)=0.022$ (Schlegel et al. 1998). The limit on ISP arising in the foreground is $0.2 \%$.

The reddening toward SN 2005hk can be estimated, if we assume that the narrow Na I D absorption component arises in the ISM of the host. The equivalent width of the Na I D absorption feature at the first epoch was measured to be $W_{\lambda}=0.30 \pm 0.02$. This value is smaller than the value reported by Chornock et al. (2006), for observations four days prior to our first observations, although the difference between the two measured values is $\sim 2 \sigma$. We note that Chornock et al. do not quote errors on their determined equivalent width. It is not clear if this difference is within the measurement errors or if it reflects evolution triggered by the SN (Patat et al. 2007). We follow Chornock et al. (2006), who adopt the results of Munari \& Zwitter (1997) for evaluating the relationship between Na I D absorption and the reddening. This relationship is based on Galactic-type extinction laws (e.g., Cardelli et al. 1989). It is,

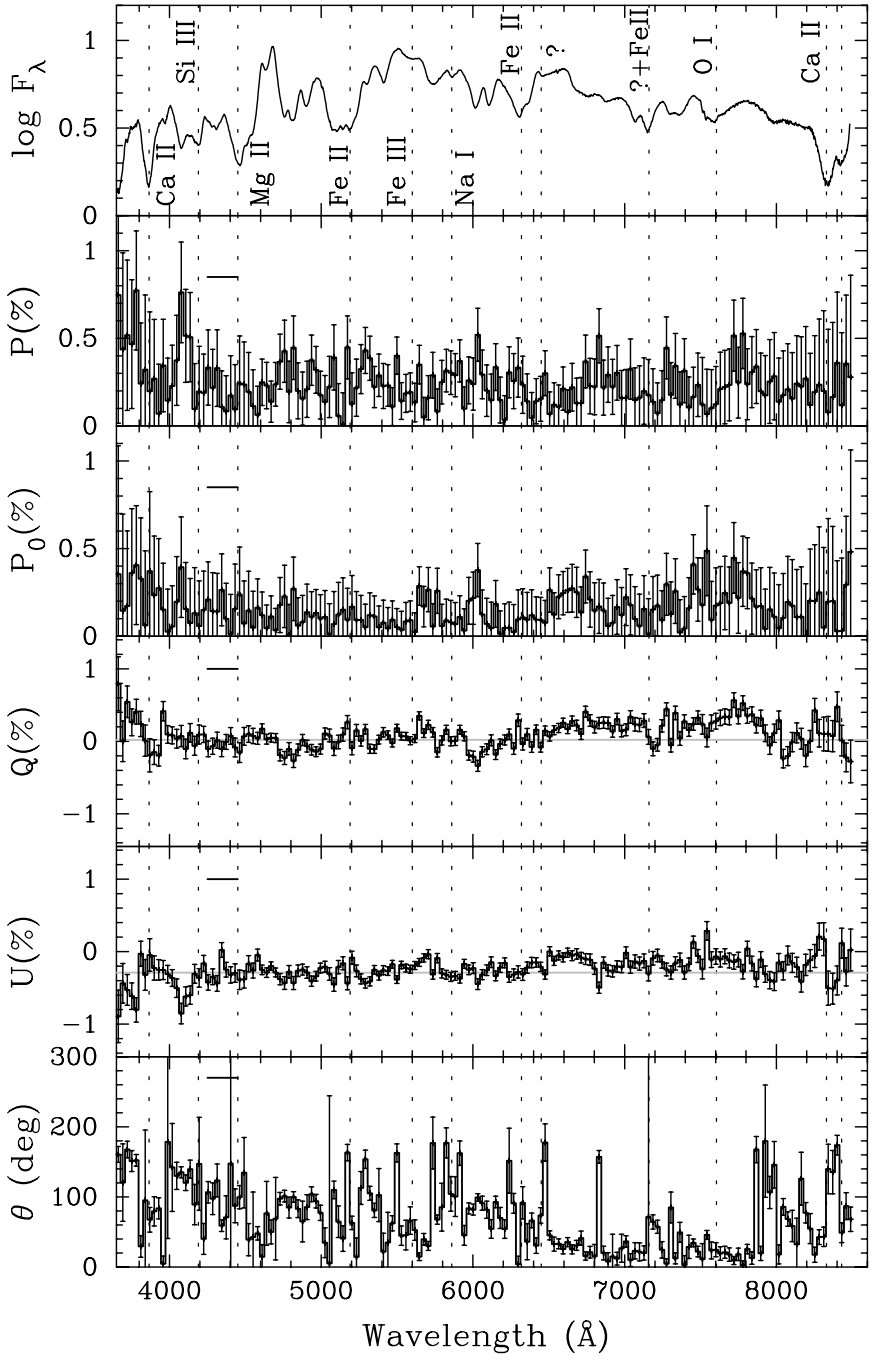

Figure 4. Same as Figure 3, but for spectropolarimetry of SN 2005hk on 2005 November 23.

therefore, implicitly assumed that the reddening component, measured from the narrow Na I absorption component in the host galaxy, follows a similar law. As the Na I D component lines are not resolved in our data, we also adopt a range of flux ratios between Na I D1 and D2 of 1.1-2 (Chornock et al. 2006). This limits the reddening of the host to $0.05<E(B-V)<0.07$. The total reddening, for Galactic-type reddening and ISP law, yields a constraint on the total ISP of $p_{\text {ISP }}<0.8 \%$.

Howell et al. (2001) present a technique for the determination of the ISP for specific wavelength ranges which have null intrinsic polarization. This assumes that, for these specific wavelength ranges, there is sufficient overlap between groups of lines such that there is complete intrinsic depolarization over these wavelengths. Howell et al. suggest that the wavelength region of 4800-5600 $\AA$ is intrinsically depolarized, where line blanketing opacity dominates over electron scattering opacity. Any polarization, observed over this wavelength range, is expected to be due to the ISP alone. For SN 2005hk, at both epochs, the lack of significant overlap and blending of lines leads to the complex behavior of the Stokes parameters. Chornock et al. (2006) find that the wavelength range of 4000-4200 A satisfies the criteria for intrinsic depolarization, namely significant line blanketing. The observation of Chornock et al. at November 5.4 


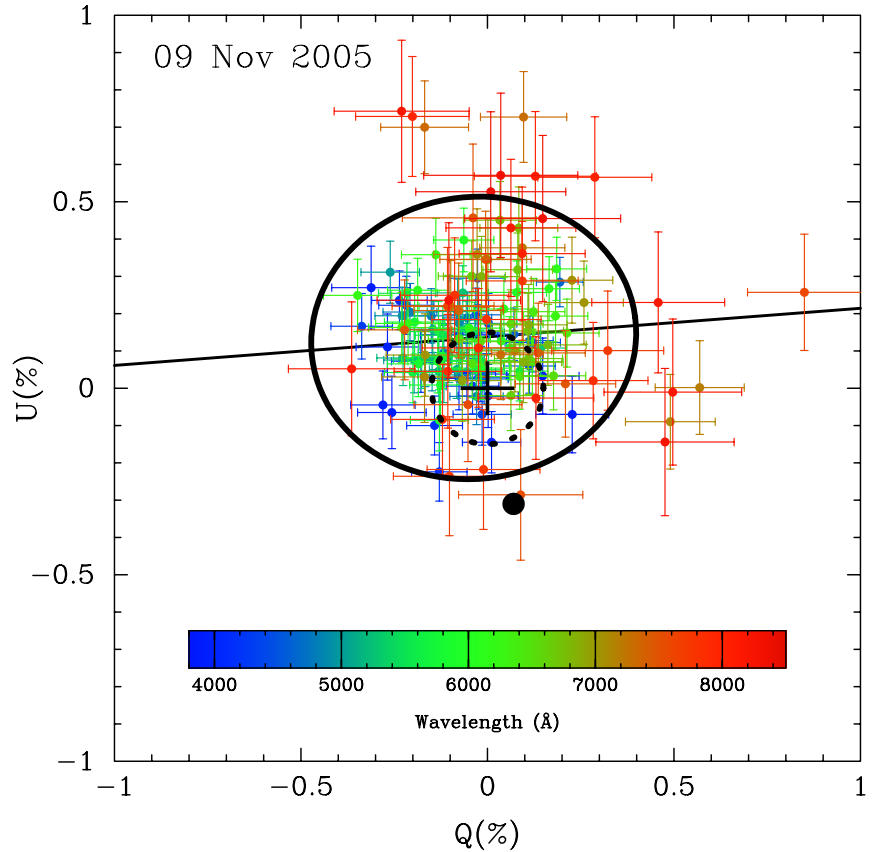

Figure 5. Spectropolarimetry of SN 2005hk on 2005 November 9, on the Stokes $Q-U$ plane. The data have been binned to $30 \AA$ for clarity, and have been corrected for the ISP component, which is indicated on the Stokes plane by the filled black circle, with the radius of the circle corresponding the measured uncertainties. The data are color coded according to wavelength following the scheme of the color bar. The origin of the Stokes plane is indicated by the cross (+). The black dashed circle represents the null polarization envelope, at $6000 \AA$. The heavy black line indicates the best fit to the "dominant axis." The ellipse shows the apparent axial ratio and orientation of the data, as determined using the PCA, with the size of the ellipse arbitrarily scaled.

(A color version of this figure is available in the online journal.)

is sufficiently close to our observation at the first epoch that we observed similar features in the polarization spectrum and the Stokes parameter spectra. Importantly, we too determine that this wavelength range provides the best approximation to a line blanketed regime. The measured Stokes parameters across this range at the first epoch are $Q=0.04 \% \pm 0.03 \%$ and $U=-0.32 \% \pm 0.03 \%$. The uncertainties on these quantities are the combined systematic and statistical uncertainties.

The spectral evolution of SN 2005hk by the second epoch, with the increase in the strengths of individual $\mathrm{Mg}$ II and Fe III lines in the range of 4000-4200 $\AA$, leads to the presence of intrinsic polarization $(Q=0.12 \% \pm 0.06 \%$ and $U=-0.47 \% \pm$ $0.060 \%$ ). Instead, we identify the region of $4250-4450 \AA$ as being spectroscopically similar to a line blanketed regime with no unblended line structure. Over this wavelength range the average values of the Stokes parameters are measured as $Q=0.09 \% \pm 0.03 \%$ and $U=-0.30 \% \pm 0.03 \%$. The measured Stokes parameters, over the depolarized wavelength range, agree with those determined at the first epoch.

We adopt values of the ISP Stokes parameters of $Q_{\text {ISP }}=$ $0.07 \% \pm 0.03 I S P$ and $U_{\text {ISP }}=-0.31 \% \pm 0.03 \%$, for both the Galactic and host ISP components, which are approximately consistent with the values measured by Chornock et al. (who calculate separate Galactic and host components). The value of the ISP relative to the observed Stokes parameters is shown in Figures 3 and 4 . These values correspond to a polarization of $p_{\text {ISP }}=0.32 \% \pm 0.03 \%$ with an angle of $\theta_{\text {ISP }}=141^{\circ} \pm 3^{\circ}$. The orientation of the ISP component is shown in Figure 1. As previously observed for other SNe (Maund et al. 2007b; Patat

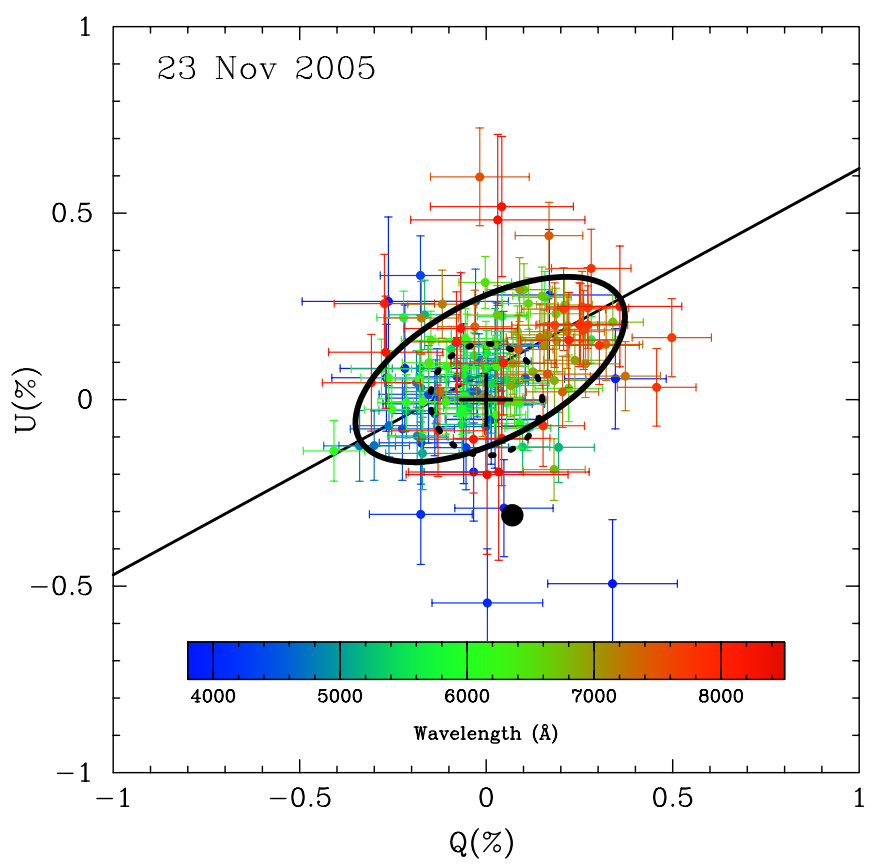

Figure 6. Same as Figure 5, but for spectropolarimetry of SN 2005hk on 2005 November 23

(A color version of this figure is available in the online journal.)

et al. 2009, 2010), the ISP component is aligned with the spiral arm pattern at the SN location, due to alignment of magnetic field lines and, hence, dust grains along spiral arms (Scarrott et al. 1987).

Given the limited wavelength range over which it can be determined, it is not clear if and how the ISP component varies with wavelength. Although a standard Serkowski-type law could be applied, it is unclear what the appropriate values of $\lambda_{\max }$ and $p_{\max }$ would be. Furthermore, it has been observed for a number of $\mathrm{SNe}$ with significant ISPs that the ISP does not necessarily even follow a Galactic-Serkowski type law of wavelength dependence (e.g., SN2006X; Patat et al. 2009). Given the small value of the ISP, we subtract, therefore, a constant ISP component from the observed data, to recover the intrinsic polarization of the $\mathrm{SN}$ (as shown by $P_{0}$ in Figures 3 and 4), under the assumption that any wavelength dependence of the ISP is smaller than the uncertainties derived above.

On the Stokes $Q-U$ plane (see Figures 5 and 6), the ISP component is located close to the centroid of the SN 2005hk data. The calculated weighted centroids for the ISP-corrected data $(\bar{Q}, \bar{U})$ are listed in Table 3 .

After correction for the ISP, the intrinsic polarization of SN 2005hk is observed to be extremely low across the observed wavelength range. The degree of polarization of a number of features observed to be relatively strongly polarized in the uncorrected polarization spectrum, is no longer as polarized once the data have been corrected for the ISP. Utilising the data across the range 6600-7000 $\AA$ as a representative of a region of pure continuum emission, the level of the continuum polarization is measured as $0.17 \% \pm 0.05 \%$ and $0.19 \% \pm 0.05 \%$ at the first and second epochs, respectively. At the second epoch the assumption that this wavelength range represents a true continuum region is most likely invalid, due to the presence of a number of minor, broad undulations which are most likely due to the lines (rather than incorrect flat fielding of the flux spectrum). This wavelength range avoids any polarization associated with 
Table 2

Polarization Changes Across Spectral Features

\begin{tabular}{lccc}
\hline \hline \multicolumn{1}{c}{ Species } & $\lambda_{\text {cont }}(\AA)$ & $\lambda_{\text {line }}(\AA)$ & $\Delta P$ \\
\hline Ca II H \& K & \multicolumn{3}{c}{2005 Nov 09} \\
Fe II & 3780 & 3960 & $0.64 \% \pm 0.15 \%$ \\
Si II & 4225 & 4315 & $0.34 \% \pm 0.11 \%$ \\
Fe II? & 6120 & 6270 & $0.54 \% \pm 0.12 \%$ \\
Ca II IR3 & 7070 & 7215 & $0.45 \% \pm 0.17 \%$ \\
& 8340 & 8490 & $0.31 \% \pm 0.40 \%$ \\
\hline Ca II H \& K & 3750 & 3930 & \\
Fe II & 4017 & 4077 & $0.49 \% \pm 0.23 \%$ \\
Fe II & 4610 & Nov 23 & $0.54 \% \pm 0.19 \%$ \\
Fe II & 6910 & $0.35 \% \pm 0.12 \%$ \\
Fe II? & 5910 & 7220 & $0.5 \% \pm 0.11 \%$ \\
& 7130 & & $0.34 \% \pm 0.14 \%$ \\
\hline
\end{tabular}

the feature at $7200 \AA$ and is less than the continuum polarization measured by Chornock et al. (2006) at 2005 November 5.4 of $0.36 \%$. This level of polarization is consistent with a spheroidal configuration with axial ratio $>0.9$ (Höflich 1991).

The significance of the polarization signal is given by $(\mathrm{S} / \mathrm{N})_{P}=P / \sigma_{P}=\sqrt{N / 2} P(\mathrm{~S} / \mathrm{N})$ (Patat \& Romaniello 2006). At both epochs there are no features, at either $15 \AA$ or $30 \AA$ binning, which are detected in the intrinsic polarization spectrum $P_{0}$ at $>3 \sigma$ and only a small number of bins detected at $>2 \sigma$. Chornock et al. (2006) argue that in their data from November 5, only the line of Fe III $\lambda 5129$ is polarized and at a rather small level. On inspection, their data suggest that Fe III $\lambda 4404$ is also somewhat polarized. There is another feature of similar amplitude showing a minimum at $\lambda 4800$ in the polarized flux that has no strong component in the total flux spectrum, but is likely to correspond to Si II $\lambda 5051$.

Our data at the first epoch also show possible modulation in the polarization spectrum associated with Fe III $\lambda 5129$, as well as Si II $\lambda 6355$ and the $7200 \AA$ feature. At redder wavelengths there are a number of peaks which are associated with telluric features, suggesting that these were variable over the course of the observations. The apparent significance of these features is therefore overestimated, as the possible variability of telluric features was not considered in the reduction process and not accounted for in the final error analysis.

By the second epoch, there are possible peaks in the intrinsic polarization associated with the absorptions of the $\mathrm{Fe}$ II multiplet 42 , the intrinsic Na I feature, and the absorption of Fe II $\lambda 6147$.

The absence of a significant signal in the polarization spectrum does not necessarily imply that the intrinsic polarization of spectral features is also not significant. Wang et al. (1997) and Leonard et al. (2002) studied the change in polarization $\Delta P$ across particular spectral features, by comparing the modulation in the Stokes $Q$ and $U$ parameters from the approximate "continuum" level and at the wavelength of absorption minimum, where

$$
\Delta P=\sqrt{\left(Q\left(\lambda_{\text {cont }}\right)-Q\left(\lambda_{\text {line }}\right)\right)^{2}+\left(U\left(\lambda_{\text {cont }}\right)-U\left(\lambda_{\text {line }}\right)\right)^{2}}
$$

$\Delta P$ is independent of the choice of the ISP component. The values of $\Delta P$ for particular spectral features, at both epochs, are presented in Table 2.

Even when assessed by modulation of the Stokes parameters, the data at both epochs show few significant features. At the first epoch, Ca II H\&K, Fe II, and, importantly, Si II $\lambda 6355$ show significant modulation, whereas at the second epoch the significant modulation is associated with the, now stronger, Fe II lines. The interpretation of the data presented in Table 2 requires some caution, however, as the selection of $\lambda_{\text {cont }}$ and $\lambda_{\text {line }}$, by eye, can lead to a bias in favor of selecting those wavelengths that yield the maximum change in the Stokes parameters. The principal differences between the data presented by Chornock et al. at -4 days, compared to our data at 0 days, are due to much larger $\mathrm{S} / \mathrm{N}$ achieved in their observation. We estimate that at $7200 \AA$, at $50 \AA$ binning, Chornock et al. achieved a final $\mathrm{S} / \mathrm{N} \approx 1400$, whereas our observation reached $\mathrm{S} / \mathrm{N} \approx 390$.

At the first epoch, two sets of two contiguous bins (at $30 \AA$ ) are observed at both the $7200 \AA$ feature and immediately redward at $7315 \AA$. An obvious line feature is associated with the former spectral line in the flux spectrum. The latter feature corresponds to a telluric feature that, although it should be equally canceled between the two beams at each retarder plate position, may appear polarized due to variability in the telluric line between the pair of observations used to derive each Stokes parameter.

On the Stokes $Q-U$ plane (see Figures 5 and 6), the data at both epochs assume an approximate elliptical distribution, with no major structure deviating from the main concentration of points. At the first epoch, the data have no obvious preferred direction. By the second epoch the data are more extended across the $Q-U$ plane, although again with no major deviations from the main concentration of data points. From Figure 6 it is also evident that, by the second epoch, the degree of polarization has become more wavelength dependent, with data at bluer wavelengths being concentrated at lower values of $Q$ and $U$ than the data at the red extreme of the observed wavelength range. Our lower $\mathrm{S} / \mathrm{N}$ data do not show the wavelength-dependent elongation to the same degree as observed at -4 days, for which a dominant axis is clear to the eye. The behavior of the data on the Stokes $Q-U$ plane, at both epochs, is quantified and discussed in Section 3.3.

\subsection{Decomposition of the Stokes Parameters}

The determination of the dominant axis, and the decomposition of the Stokes vector, permits a correction for the random P.A. at which target $\mathrm{SNe}$ have been observed, as well as determination of the principal axis of symmetry of the data, the relative orientations of the different line-forming species and, ultimately, the underlying geometry. The dominant axis forms a line across the Stokes $Q-U$ plane, and the decomposition process involves rotating the data so that the principal rotated Stokes axis is aligned with the axis of greatest variance for the data on the Stokes plane. The polarization signal is measured in the two orthogonal directions, which are effectively the "rotated Stokes parameters," along the dominant axis $\left(P_{d}\right)$ and along the orthogonal axis $\left(P_{o}\right)$. A polarization component in the orthogonal direction permits the identification of deviations from the single axial symmetry represented by the dominant axis.

Here, we explore two complementary techniques for determining the dominant axis: least-squares fitting of a straight line and a principal components analysis of the data on the Stokes $Q-U$ plane. Importantly, these two different analyses should give identical measures of the dominant axis, but provide different parameterization of the data on the Stokes $Q-U$ plane. This latter point is particularly crucial for applying the SN spectropolarimetric classification scheme of Wang \& Wheeler (2008). 
Table 3

Best-fit Dominant Axes

\begin{tabular}{|c|c|c|c|c|c|c|c|c|}
\hline \multirow[t]{2}{*}{ Date } & \multicolumn{4}{|c|}{ Least Squares Fit Analysis } & \multicolumn{2}{|c|}{$\begin{array}{c}\text { Principal } \\
\text { Components }\end{array}$} & \multicolumn{2}{|c|}{ Centroids } \\
\hline & $\alpha$ & $\beta$ & $\chi^{2} /$ dof & $\theta_{\text {dom }}$ & $\begin{array}{c}\text { Axial Ratio } \\
b / a\end{array}$ & $\theta_{e} / 2$ & $\bar{Q}$ & $\bar{U}$ \\
\hline $2005 \operatorname{Nov} 9^{a}$ & $0.138 \pm 0.008$ & $0.077 \pm 0.074$ & $347.2 / 154$ & $2.2 \pm 7.7$ & 0.87 & 3.7 & $-0.037 \pm 0.016$ & $0.135 \pm 0.016$ \\
\hline 2005 Nov $9^{b}$ & $0.138 \pm 0.009$ & $0.226 \pm 0.084$ & $202.3 / 98$ & $6.4 \pm 7.9$ & 0.78 & 8.7 & $-0.049 \pm 0.016$ & $0.127 \pm 0.016$ \\
\hline $2005 \operatorname{Nov} 23^{a}$ & $0.075 \pm 0.008$ & $0.545 \pm 0.051$ & $288.8 / 154$ & $14.3 \pm 5.0$ & 0.47 & 14.0 & $0.011 \pm 0.016$ & $0.081 \pm 0.015$ \\
\hline $2005 \operatorname{Nov} 23^{\mathrm{a}}$ & $0.079 \pm 0.010$ & $0.771 \pm 0.077$ & $160.9 / 98$ & $18.8 \pm 5.0$ & 0.42 & 18.55 & $-0.022 \pm 0.016$ & $0.062 \pm 0.016$ \\
\hline
\end{tabular}

Notes.

${ }^{\text {a }}$ For data over the wavelength range $3700-8600 \AA$.

${ }^{b}$ For data over the wavelength range 4000-7000 ̊.

\subsubsection{Least-squares Fit to the Data}

Straight lines, of the form $U=\alpha+\beta Q$, were fit to the data of both epochs, using a weighted least-squares fitting technique, where the data were weighted according to the errors in both $Q$ and $U$ (Press et al. 1992). In order to confirm the validity of this technique, the form of the straight line was reversed and the best fit was calculated for $Q=1 / \beta U-\alpha / \beta$, giving identical results. The value of $\beta$ yields the angle of the dominant axis with respect to the Stokes $Q$-axis, through which the data are to be rotated to decompose the Stokes vector onto the dominant and orthogonal axes. For a single axial symmetry, the dominant axis will pass through the origin of the Stokes $Q-U$ plane. In instances where the data are offset from the origin (e.g., by a wavelength-independent continuum polarization component) the offset will be partially manifested in the measured $\alpha$ parameter.

Dominant axes were calculated for the data at both epochs over two different wavelength ranges: $3700-8600 \AA$ and 4000-7000 $\AA$. The former range wavelength encompasses the entire wavelength range of the observed data, whereas the latter excludes regimes with poor levels of signal to noise $(\mathrm{S} / \mathrm{N} \leqslant 200)$. The regions of low $\mathrm{S} / \mathrm{N}$ arise at the blue and red extremes of the data, due to line blanketing and the blue spectral energy distribution significantly reducing the observed flux in the blue and red, respectively, and the declining detector response (in the red).

The results of the least-squares analysis and the properties of the determined dominant axes are presented in Table 3. The values of $\alpha, \beta$, and the polarization angle of the dominant axis $\left(\theta_{\mathrm{dom}}=\frac{1}{2} \arctan \beta\right)$ were found to be relatively insensitive, within the uncertainties, to the choice of the wavelength range over which the dominant axis was calculated. The dominant axes are shown on the Stokes $Q-U$ plane in Figures 5 and 6 . The large values of $\chi^{2}$ for the straight line fit, at both epochs, indicate that the data are poorly described by a straight line, with a significant polarization component in the orthogonal direction. At the first epoch, simple inspection of the data on the Stokes $Q-U$ plane would suggest that the data are evenly distributed in angle about the centroid (as is illustrated in Section 3.3.2). At the second epoch, a dominant axis is more clearly observed.

\subsubsection{Principal Component Analysis}

In order to better understand and quantify the distribution of the data on the $Q-U$ Stokes plane and, in particular, the degree of the orthogonal polarization component, a principal component analysis of the data was conducted.
The elements of the $2 \times 2$ weighted covariance matrix take the form:

$$
\operatorname{cov}_{w}\left(X_{j}, X_{k}\right)=\frac{\sum_{i} \frac{1}{\Delta X_{i j}} \frac{1}{\Delta X_{i k}}\left(X_{i j}-\bar{X}_{j}\right)\left(X_{i k}-\bar{X}_{k}\right)}{\sum_{i} \frac{1}{\Delta X_{i j}} \frac{1}{\Delta X_{i k}}} \frac{N}{N-1}
$$

where $j, k=0,1, X_{0}=Q, X_{1}=U$ (see Bevington \& Robinson 2003), $i=1, \ldots, N$ and the weighted average of the Stokes parameters are $\bar{X}_{j}=\sum_{i} \frac{X_{i j}}{\Delta X_{i j}^{2}} / \sum_{i} \frac{1}{\Delta X_{i j}^{2}}$, as shown in Table 3, over the $N$ wavelength bins. The eigenvectors and eigenvalues of the covariance matrix provide an alternative measure of the angles of the dominant and orthogonal axes $\left(\theta_{e} / 2\right)$ and the ratio of the degrees of polarization in the dominant and orthogonal directions (an axial ratio for the resulting ellipse $b / a)$, respectively. This latter quantity can be used to describe an ellipse on the Stokes $Q-U$ plane which contains the data. The results of the principal component analysis are presented in Table 3, and corresponding ellipses are presented in Figures 5 and 6 . As expected, the polarization angle of the dominant axis determined using this analysis agrees, within the uncertainties, with the result from the least-squares fit.

The non-zero values of $b / a$ determined from the principal components analysis show that the data at the two epochs are inconsistent with both straight lines and null polarization. This suggests that, although the polarization measured at both epochs is low, we have measured a real polarization signal for $\mathrm{SN}$ 2005hk. The ratio of the dominant and orthogonal components measured at the second epoch is smaller than that measured at the first, implying that at the first epoch the ejecta were approximately round and, with time, departed more significantly from the spherical symmetry.

This interpretation requires some caution, however, as Chornock et al. observed data that corresponded to a dominant axis four days prior to our first observation, which we do not see in our data at the first epoch. This may reflect the low S/N of our first observation, rather than an "oscillating" evolution in shape of the structure of the ejecta; with the ejecta appearing approximately spherical at maximum but departing from a spherical symmetry both before and after maximum light.

\subsubsection{The Data on the Dominant and Orthogonal Axes}

The data were rotated onto the dominant and orthogonal axes, using the angles determined from the least-squares fit analysis (for the limited wavelength range of 4000-7000 $\AA$ ). The rotated data are presented in Figures 7 and 8. Despite the apparent increase in the polarization angle from the first to second epochs, the determined polarization angles for the 


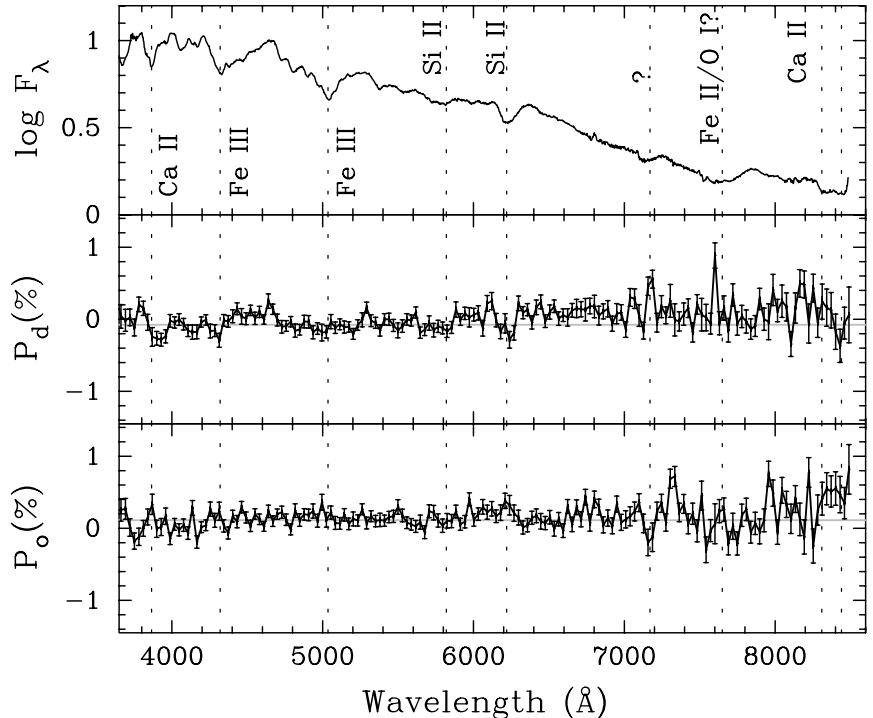

Figure 7. Spectropolarimetry of SN 2005hk on 2005 November 9, projected onto the dominant axis $\left(P_{d}\right)$ and the orthogonal axis $\left(P_{o}\right)$. The data have been binned to $30 \AA$ for clarity and have been corrected for the ISP component. The weighted average of the ISP, rotated onto the dominant and orthogonal axes, is indicated by the heavy gray lines.

dominant axes are different from the angle measured at -4 days, for which Chornock et al. determined $\theta_{\text {dom }}=35^{\circ} .6$.

At the first epoch, the dominant and orthogonal polarization components are similar to Stokes $Q$ and $U$, due to the small angle through which the data were rotated. The polarization modulations associated with Ca II, Fe III, and Si II are predominantly observed in the dominant polarization component, and over the range 4000-7000 $\AA$ the deviations observed in the orthogonal polarization spectrum are consistent with the expected noise level. Similar to the Chornock et al. observations at -4 days, an important exception is the $7200 \AA$ absorption feature (and possibly Fe III $\lambda$ 5129) which has both dominant and orthogonal polarization components (although Chornock et al. refer to the rotated Stokes parameters $q_{\mathrm{RSP}}$ and $u_{\mathrm{RSP}}$, these are equivalent to our dominant and orthogonal axes). At our second epoch, the majority of features, associated with Fe II, Na I and, particularly, the $7200 \AA$ feature show some modulation in both the dominant and orthogonal polarization components. While Chornock et al. observed an obvious wavelength dependence in the dominant polarization component, with increasing $P_{d}$ at redder wavelengths (which we estimate to be $0.1 \%$ per $1000 \AA$ ), four days later we found the wavelength dependence to be shallower $0.05 \% \pm 0.01$ per $1000 \AA$. At +14 days we measure a similar wavelength dependence $0.07 \% \pm 0.01$ per $1000 \AA$. This wavelength dependence is clearly seen in Figure 6. The change may not be evidence for evolution in the wavelength dependence of the dominant polarization component, in itself, as the observations of Chornock et al. were acquired with higher S/N. Such wavelength dependence of the polarization, increasing at redder wavelengths was observed for SN 1999by (Howell et al. 2001). At the temperatures determined for SN 2005hk, and for Fe-rich matter, the opacity at the photosphere is dominated by blends of line transitions for $\lambda<6000 \AA$ (see Figure 2 of Höflich et al. 1993). At redder wavelengths, the opacity is primarily due to electron scattering. The continuum polarization may indicate an aspherical Thomson scattering region, e.g., by a global asymmetry induced by the rotation of the white dwarf progenitor (Howell et al. 2001) or off-center delayed detonations (Höflich

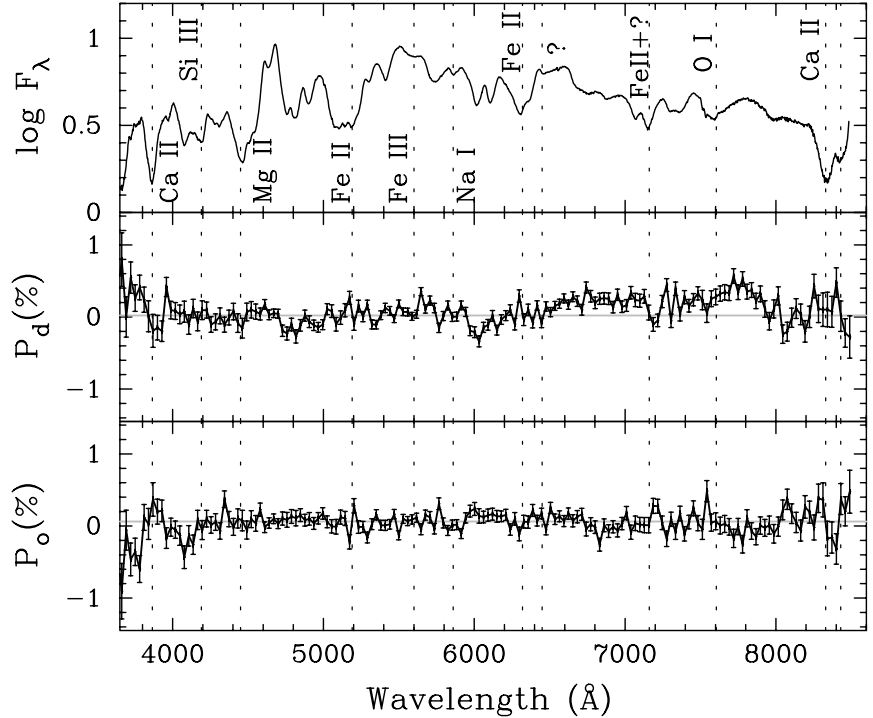

Figure 8. Same as Figure 7, but for spectropolarimetry of SN 2005hk on 2005 November 23 .

et al. 2006). Because multiple line scatterings are effectively depolarizing, the observation of increasing polarization with wavelength may be understood in terms of the decrease of the role of line opacity at redder wavelengths. If the dominant axis corresponds to a principal axial symmetry, then the line-forming regions of the species observed in the flux spectrum follow the same axial symmetry as that of the continuum at the first epoch, but not at the second epoch.

\subsection{The Inferred Ejecta Geometry}

On the basis of the continuum polarizations measured at 0 and 14 days, any asymmetry in the shape of the photosphere (assuming a spheroidal configuration) requires an axial ratio $>0.9$ (Höflich 1991). A similar limit on the asphericity of the photosphere is achieved if one uses the higher value of continuum polarization $(0.36 \%)$ determined by Chornock et al.

The general level of line polarization is low, suggesting that the line-forming regions cover an approximately spherically symmetric photosphere homogeneously. Limits on the amount of polarization expected to be observed can be calculated, given a level of continuum polarization and line depth, under the assumption that the line-forming region blocks only unpolarized light from the photosphere, while not blocking polarized light arising from the limb (i.e., that the photosphere can be separated into discrete polarized and unpolarized zones):

$$
p_{\text {line }} \leqslant p_{\text {cont }} \cdot \frac{I_{\text {cont }}}{I_{\text {line }}},
$$

where $I_{\text {cont }}$ and $I_{\text {line }}$ are the observed fluxes in the continuum and in the absorption line minimum, respectively (i.e., such that the line depth is $I_{\text {cont }}-I_{\text {line }}$ - this convention is chosen as the observed polarization arises from the light that is not absorbed). As Chornock et al. (2006) calculated for their observation of SN 2005hk, we also calculate the theoretical limits on the line polarization for measured absorption line depths in our observations.

At the first epoch, the maximum polarization for the Si II $\lambda 6355$ line is only $0.14 \%$. As Chornock et al. suggest, that the lines are not strongly polarized is commensurate with their apparent weakness. This is true at the first epoch, where line 
strengths of only $>0.7$ are observed. At the second epoch, however, absorption line depths of $\sim 0.5$ are observed, and with the increase in depth of these lines it might be expected that, should the line-forming regions be asymmetric, there would also be a commensurate increase in the polarization observed across these lines (see Figure 9).

Equation (3) assumes that all of the polarization is due to the continuum, which implicitly assumes that the photosphere itself is asymmetric. In a number of cases, in particular for core-collapse $\mathrm{SNe}$, the classical limit for line polarization is exceeded (see e.g., Maund et al. 2007a, 2009), for low values of continuum polarization which suggest approximately spherical photospheres. Violations of this criterion can be explained by large-scale structure, with a dissimilar geometry to that of the photosphere, or clumping, which may produce polarization irrespective of the shape of the photosphere or the degree of continuum polarization. The degree of observed polarization, for a clumpy line-forming region, should depend on the size of the clumps: for a given line absorption depth, fewer larger clumps block larger areas with contiguous polarization vectors, whereas more smaller clumps can be distributed more evenly across the photosphere.

In order to explore the possible role of clumping, and the expectation for the production of polarization across shallow lines, a Monte Carlo toy model of a simple photosphere and obscuring clumped line-forming region was constructed following the scheme of Wang et al. (2007). A circular continuum emitting photosphere was adopted, with the luminosity as a function of projected radius $r_{p}$ across a photosphere of radius $R_{\mathrm{ph}}$ set to

$$
I\left(r_{p}\right) / I(0)=1-k\left(1-\sqrt{1-\left(r_{p} / R_{\mathrm{ph}}\right)^{2}}\right)
$$

with limb darkening modeled using the coefficient $k$, which for $k=0$ yields no limb darkening. For a three-dimensional spherical photosphere, this would correspond to a limb darkening law of $I\left(r_{p}=R_{\mathrm{ph}} \sin \theta\right) / I(0)=(1-k(1-\cos \theta))$. The radial dependence of the polarization was assumed to follow a quadratic form $p \propto\left(r_{p} / R_{\mathrm{ph}}\right)^{2}$, with the constant of proportionality chosen to yield $15 \%$ polarization for light emitted at the limb of the photosphere (Chandrasekhar 1960). Opaque circular clumps were assumed, with $\tau \gg 1$, such that all photospheric flux from the region underlying the clump was blocked. For each iteration of the model, $N$ clumps of size $R_{\text {clump }} / R_{\mathrm{ph}}$ were randomly positioned across the photosphere and the corresponding flux of the uncovered regions, and their polarization components, were summed. Clumps were permitted to overlap and only partially cover the photosphere. For each size of clumps, 3000 simulations were conducted. This permits a comparison of the expected polarization for a given absorption line depth, assuming a particular clump size in the line-forming region. The results of this model (for $R_{\text {clump }} / R,=0.1,0.2,0.3$ and 0.4 ) are shown in Figure 9.

For some of the models, zero polarization was achieved for uniform coverage of the photosphere by the clumps, however, the difference between the average polarizations expected (as shown in Figure 9) and null polarization was $>1 \sigma$ for all the calculated models. These models suggest that, for a clumpy line-forming region, the clump scale for SN $2005 \mathrm{hk}$ is very small $\left(\leqslant 0.2 R_{\mathrm{ph}}\right)$ to produce lines of the depths observed, in the flux spectra at both epochs, with low polarization. The uniformly low polarization observed for lines arising from different species suggests that the ejecta are generally mixed, with no separate structures on the plane of the sky for different elements or

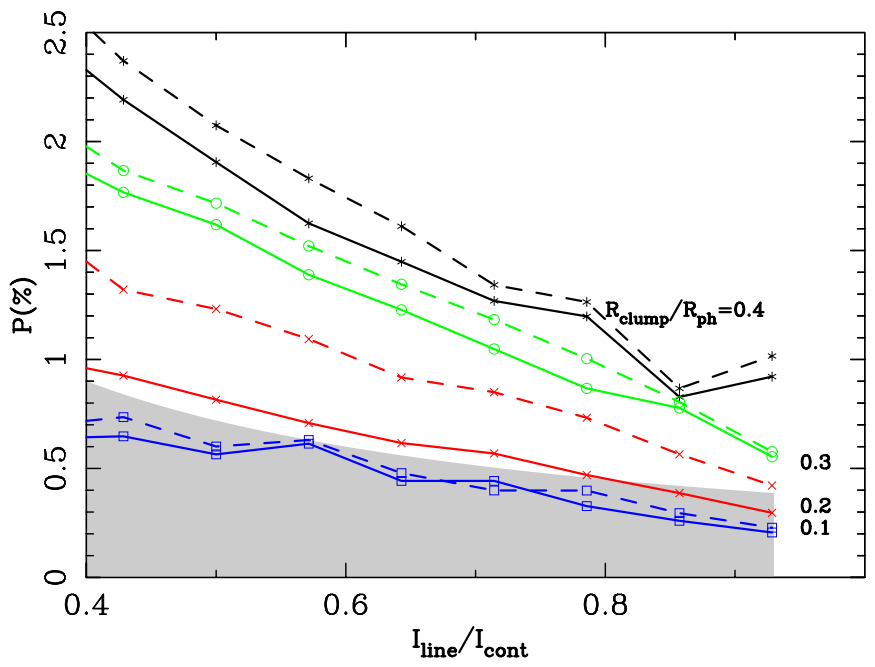

Figure 9. Results of Monte Carlo simulations of random obscuring spherical clumps in front of a spherical photosphere. The models are shown for clump scales of $R_{\text {clump }} / R_{,}=0.1$ (blue $\square$ ), 0.2 (red $\times$ ), 0.3 (green $\circ$ ), and 0.4 (black $*$ ). Solid lines are for simulations with $k=0.5$, while dashed lines indicate simulations with $k=0$. The shaded area indicates the permitted values of line polarization, according to Equation (3), for a continuum polarization of $0.36 \%$ determined by Chornock et al. (2006). Lower values of the continuum polarization $(0.17 \%$ determined here) place even stricter upper limits on the scale of clumping. Minor fluctuations in the curves are due to the statistical nature of the Monte Carlo simulations; except for the case of $R_{\text {clump }} / R_{\mathrm{ph}}=0.4$ for $I_{\text {line }} / I_{\text {cont }}>0.8$, where the results are dominated by the finite size of the "pixels" that compose the model photosphere.

(A color version of this figure is available in the online journal.)

ionization stages(e.g., Fe II and Fe III). The element responsible for the absorption at $7200 \AA$ may be an exception, with a lineforming region that is more aspherical than other species. The data for SN 2005hk, at the two epochs presented here and those presented by Chornock et al. (2006), are consistent with an approximately spherical symmetric photosphere surrounded by similar line-forming regions for all species.

The principal assumption of the model presented above is that the line-forming region and the photosphere are separate. As discussed in Section 3.3.3, the opacity at the photosphere (where the quasi-continuum forms) is not due to electron scattering alone, but rather includes a significant wavelength-dependent contribution from atomic transitions; this implies that lineforming regions are not, in reality, separate entities except in the most high-velocity regions such as those discussed by Kasen et al. (2003).

The size of the clumps and their relation to "large-scale" structure within the ejecta, leads to an interesting problem of semantics: since very large clumps $\left(R_{\text {clump }} / R_{\mathrm{ph}}=0.4\right)$ may be considered large-scale structure and very small clumps $\left(R_{\text {clump }} / R_{\text {ph }}=0.1\right)$ tend toward constituting an effectively homogeneous medium. Large opacities require that a large fraction of the photosphere, the last Thomson scattering distance, are reprocessed by lines, mostly due to iron-group elements (which will result in a significantly altered spectrum).

\section{DISCUSSION}

\subsection{SN 2005hk as a Type Ia SN}

The class of SNe similar to SN 2002cx currently has 15 members, including 2005hk and 2008ha (Foley et al. 2009). As discussed in detail by $\mathrm{Li}$ et al. (2003) and Phillips et al. (2007), both SN 2002cx and SN 2005hk were of especially low 
velocity at the photosphere near maximum light, $\sim 6000 \mathrm{~km} \mathrm{~s}^{-1}$, had early relatively high ionization, and low peak luminosity. Neither showed the typical secondary maxima in $R$ and $I$ of normal SNe Ia, but were also dissimilar to previously studied under-luminous SNe Ia that do not show the secondary maxima (e.g., Filippenko et al. 1992). There is also extreme diversity within the class, with SN 2008ha being $\approx 3$ mag fainter than SN 2002cx (Foley et al. 2009). The light curve decline is rather slow, unlike previous under-luminous SNe Ia that serve to define the light curve brightness/decline relation prominently used in cosmology studies.

Li et al. (2003) pointed out that no published model of an SN Ia corresponds to all the behavior of SN 2002cx, but suggested that a pulsating delayed detonation (Khokhlov 1991) model might be worth considering further. Jha et al. (2006) suggested that the nebular spectral characteristics that they determined might be consistent with a pure deflagration model since the models in the literature have insufficient energy to correspond to normal $\mathrm{SNe}$ Ia and hence tend to have smaller expansion velocities and produce less ${ }^{56} \mathrm{Ni}$ and hence tend to be dim. Phillips et al. (2007) suggest that the low expansion velocities, low peak luminosity, and the absence of secondary maxima in the NIR light curves may be reproduced by a three-dimensional pure-deflagration model (Gamezo et al. 2004; Röpke et al. 2006) that produces $0.25 M_{\odot}$ of ${ }^{56} \mathrm{Ni}$.

A key issue with the hypothesis that this class of explosions represents pure deflagrations of white dwarfs is that the pure deflagration models leave a substantial amount of unburned $\mathrm{C}$ and $\mathrm{O}$ in the outer layers. This $\mathrm{C}$ and $\mathrm{O}$ should show up in the early phases and while O I $\lambda 7774$ is clearly present at the second epoch (and possibly the first), the case for $\mathrm{C}$ II $\lambda 6578$ (and possibly for a contribution to the $7200 \AA$ feature) is less clear but it is not conclusively absent. In addition, Kozma et al. (2005) suggest that the late-time spectra of pure deflagration models show no similarity to that of SN 2002cx either, in particular the absence of strong features due to $\mathrm{C}$ and O. Jha et al. (2006) claim a marginal detection of $\mathrm{O}$ I in late-time nebular spectra of SN 2002cx, with the suggestion that more unburned C and $\mathrm{O}$ may be contained inside the dense, still optically thick core at late times. Sahu et al. claim that at early times O I $\lambda 7774$ is suppressed by high temperatures and, at late times, nebular $\mathrm{O}$ I lines are also suppressed by high temperatures. The lack of observation of O I $\lambda 7774$ for SN 2005hk at very early times (before our observations) is uncertain, due to poor spectral coverage at early times (Phillips et al. 2007) and low S/N (Sahu et al. 2008). Sahu et al. (2008) are able to approximately model the photospheric spectrum of SN 2005hk, based on the W7 deflagration explosion model (Nomoto et al. 1984). Importantly, Sahu et al. find that, given the conditions of the SN, their spectral synthesis suggests large amounts of unburned $\mathrm{O}$. Their model also assumes spherical symmetry, for which they achieve a reasonable approximation to the line profiles (which are somewhat sensitive to any departures from spherical symmetry; see, e.g., Tanaka et al. 2009 for a counterexample). The features remain too broad, are forbidden rather than permitted, and show prominent emission of [O I] $\lambda 6300,6364$. High densities, as evidenced by the low expansion velocities and P Cygni profiles observed at late nebular epochs, might suppress the [O I] feature, but it is predicted in the models and so its absence is a definite constraint on the nature of SN 2005hk.

Chornock et al. (2006) consider the possibility that these events may be related to normal SNe Ia, but viewed along the "hole" left by having the binary companion nearly directly along the line of sight (Marietta et al. 2000; Kasen et al. 2004). Such an aspect might increase the prominence of high-ionization species, weaken absorption lines, reduce the photospheric velocity, and induce some polarization due to the non-spherical geometry of the ejecta. One issue with this model for SN 2002cx and related explosions is that it would result in higher luminosities than when viewed from other aspect angles. Even if the underlying explosion were a subluminous event such as SN 1991bg, if the view were "down the hole," the result would be brighter than SN 1991bg. Both SN 2002cx and SN 2005hk were subluminous by about $1 \mathrm{mag}$ in all bands (except $H$; Phillips et al. 2007). It is certainly not clear that this is consistent with a "hole" model. Chornock et al. 2006 comment that their measurement of a small but appreciable continuum polarization and weak line features was inconsistent with the prediction of Kasen et al. (2004) of a continuum polarization of $\sim 0.1 \%$ and strong line polarization peaks for an explosion viewed slightly off the axis of the hole. As the $\mathrm{SN}$ evolves, the relative size of the hole and the photosphere will change (in particular if the hole closes, which Kasen et al. 2004 expect to be a slow process if it occurs), which should lead to a commensurate change in the polarization properties. Importantly, we see only moderate change in the polarization, despite the significant changes in the flux spectrum, between the epochs at which SN 2005hk was observed. In addition, it is not at all clear how such a model would account for the late-time permitted line spectrum delineated by Jha et al. (2006). Our observations do suggest some line polarization. This demands some asymmetry in the ejecta, consistent with a single axial symmetry, but does not spell out quantitative agreement with the "hole" model.

An alternative model might be a "plume" model (Plewa et al. 2004; Livne et al. 2005) in which a single plume (or very few plumes) reaches the surface, spreading fresh ${ }^{56} \mathrm{Ni}$. If the plume burned little, the expansion might be slow and leave high densities at late times. Our spectropolarimetry suggests that the outer layers of the ejecta are approximately homogeneously mixed, although stratified, which suggests mixing in a large number of smaller plumes (with size $\leqslant 0.2 R_{\mathrm{ph}}$ at the first epoch).

This class of objects thus presents many conundra. They show high excitation early on but otherwise have none of the properties of SNe Ia like SN 1991T that show early Fe III. Their light curves are dim but broad, violating the "Phillips relation." They have very slow expansion velocities and may remain partially optically thick at nearly 300 days after the explosion. They are mildly asymmetric in the continuum, in the Fe III early on and in Si II near maximum. The polarization of SN 2005hk is completely compatible with the observed range of polarization properties for Type Ia SNe (see Table 4). The level of polarization of SN 2005hk is not the lowest detected for an SN, with SN 1996X (a normal Type Ia SN; Wang et al. 1997) showing polarization just above statistical significance and with no apparent dominant axis. The observations of SN 2005hk show it to have a spectropolarimetric type of D1 (Wang \& Wheeler 2008), similar to most Type Ia SNe, indicating that there is a principal axis of symmetry to the ejecta. Importantly, there are no apparent loop like features, even for the strongest lines (which is particularly pertinent for the observations at the second epoch) which would indicate a significant departure from this main axial symmetry. Normal Type Ia $\mathrm{SNe}$, such as SN 2001el (Wang et al. 2003b) and SN 2004S (Chornock \& Filippenko 2008), show some structure in their ejecta principally in the Si II, as well as the high velocity component of the Ca II 
Table 4

Comparison SN Spectropolarimetry

\begin{tabular}{|c|c|c|c|c|c|c|}
\hline $\mathrm{SN}$ & $\begin{array}{l}\text { Spectral } \\
\text { Type }\end{array}$ & Epoch $^{\mathrm{a}}$ & $\begin{array}{c}\text { SP } \\
\text { Type }^{\text {b }}\end{array}$ & $\begin{array}{c}\text { Continuum } \\
\text { Pol. }(\%) \\
\end{array}$ & $\begin{array}{c}\text { Line } \\
\text { Pol. }(\%)\end{array}$ & Ref. \\
\hline $1996 X$ & Ia (normal) & -7 & N1 & $<0.3$ & Si II:0.6 \pm 0.15 & 1 \\
\hline 1999by & Ia (sub) & -1 & D1 & $0.8 \pm 0.1$ & & 2 \\
\hline 2001el & Ia (normal) & +2 & $\mathrm{D} 1+\mathrm{L}(\mathrm{Ca})$ & 0.3 & Si:0.3;Ca:0.7 & 3 \\
\hline $2003 d u$ & Ia (normal) & +18 & D1 & & 0.2 & 4 \\
\hline $2004 S$ & Ia (normal) & +9 & $\mathrm{D}$ & $0.4 \%$ & $\leqslant 0.5$ & 5 \\
\hline $2004 d t$ & Ia (normal) & -7 & $\mathrm{D} 1(\mathrm{Si}+\mathrm{Mg})$ & & Si II:2 & 6 \\
\hline $2006 X$ & Ia (normal) & 0 & $\mathrm{D} 1+\mathrm{L}$ & $<0.2$ & Si II:0.8 & 7 \\
\hline $2005 \mathrm{hk}$ & 2002cx-like & -4 & D1 & 0.4 & & 8 \\
\hline$\ldots$ & $\ldots$ & 0 & D1 & $\sim 0.2$ & & this work \\
\hline$\ldots$ & $\ldots$ & 14 & D1 & $\sim 0.2$ & & this work \\
\hline 2002ap & Ic (broad-lined) & 0 & DL & $\sim 0.3 \%$ & OI:4 & 9 \\
\hline $2005 b f$ & $\mathrm{Ib}(\mathrm{pec})$ & $-6^{\mathrm{c}}$ & $\mathrm{D} 1, \mathrm{~L}(\mathrm{He}+\mathrm{Ca})$ & $<0.45 \%$ & He I:1.3,Ca II:4 & 10 \\
\hline $2007 \mathrm{gr}$ & Ic & +21 & $\mathrm{D} 1+\mathrm{L}(\mathrm{Ca})$ & $0.5 \%$ & Ca II: 2.5 & 11 \\
\hline 2008D & $\mathrm{Ib}$ & 0 & $\mathrm{D} 1+\mathrm{L}(\mathrm{He}+\mathrm{Ca})$ & $<0.4 \%$ & He I:0.6,Ca II:1.8 & 12 \\
\hline$\ldots$ & $\ldots$ & 14 & $\mathrm{D} 1+\mathrm{L}(\mathrm{He}+\mathrm{Ca})$ & $<0.4 \%$ & He I:1.1,Ca II:2.5 & 12 \\
\hline
\end{tabular}

Notes.

${ }^{\text {a }}$ Relative to optical maximum.

b SN Spectropolarimetric types see Wang \& Wheeler (2008).

c Relative to the second maximum. References: (1) Wang et al. 1997; (2) Howell et al. 2001; (3) Wang et al. 2003b; (4) Leonard et al. 2005; (5) Chornock \& Filippenko 2008; (6) Wang et al. 2006; (7) Patat et al. 2009; (8) Chornock et al. 2006; (9) Wang et al. 2003a, Kawabata et al. 2002, Leonard et al. 2002; (10) Maund et al. 2007a; (11) Tanaka et al. 2008; (12) Maund et al. 2009.

IR3 (see also Wang et al. 2003b; Patat et al. 2009). Conversely, there are Type Ia SNe, such as SN 2004dt (Wang et al. 2006), whose line polarization is principally in the dominant polarization component which is consistent with a single axial symmetry. In the case of SN 2004dt, however, the observed line polarization was $\sim 1 \%$. In summary, the similarity between the spectropolarimetry of SN $2005 \mathrm{hk}$ and Type Ia SNe indicates that their respective explosion mechanisms are both approximately spherical (see Table 4).

Wang et al. (2007) identified a correlation between the degree of polarization of $\mathrm{Si}$ II, at -5 days, with the decline rate $\left(\Delta m_{15}(B)\right)$ of the light curve. At some wavelengths SN 2005hk is an outlier from the Phillips brightness-decline relationship, although it is consistent with the relationship in the $I$ and $H$ bands (Phillips et al. 2007). While the polarization measurements of SN 2005hk are offset from the measured relation derived for normal Type Ia SNe by Wang et al., it is not as significant an outlier as the subluminous Type Ia SNe 1999by and 2005ke (see Figure 10).

\subsection{SN 2005hk as a Core-collapse SN}

Jha et al. (2006), Valenti et al. (2009), and Foley et al. (2009) consider the possibility that events such as SN 2002cx and 2005hk are not related to Type Ia SNe, but may instead be a type of CCSN. Based on the photometric and spectroscopic similarity between SN 2008ha and SN 2005hk with lowluminosity Type IIP and Type Ic SNe (2005cs and 2007gr, respectively) and the occurrence of these events in late-type galaxies, Valenti et al. (2009) suggest 2002cx-like SNe are lowluminosity Type Ic SNe, potentially associated with gammaray bursts. Foley et al. (2009) present additional events in this class, which are claimed to have taken place in lenticular S0 galaxies, which may argue against massive stars as the universal progenitors for this type of event. Spectropolarimetry provides additional parameter space in which to examine the similarities between different types of $\mathrm{SNe}$.

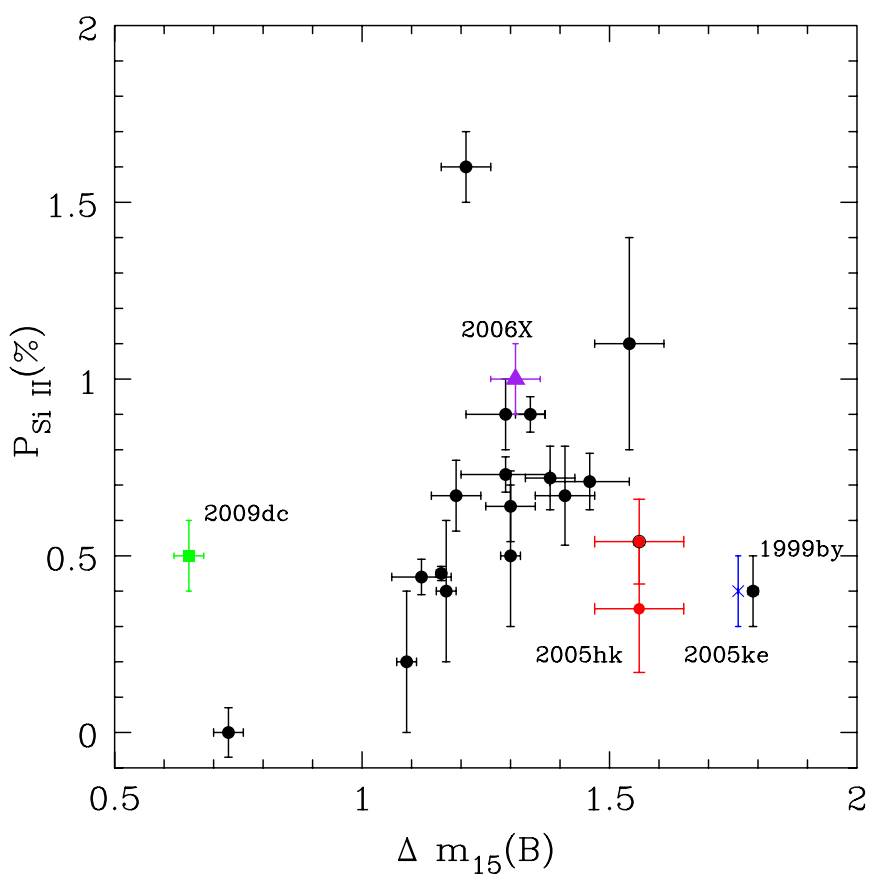

Figure 10. Degree of polarization across Si II $\lambda 6355$ as a function of light curve decline rate. Our measurements of SN 2005hk are shown in red, with the value of $\Delta P(\mathrm{Si}$ II $)$ indicated by the point with the heavy outline, data in black are taken from Wang et al. (2007), the measurement for SN 2006X $(\triangle)$ is from Patat et al. (2009), the measurement for the luminous event SN 2009dc ( $\square$ ) is taken from Tanaka et al. (2010), and the measurement for SN 2005ke $(\times)$ is taken from F. Patat et al. (2010, in preparation).

(A color version of this figure is available in the online journal.)

Valenti et al. (2009) and Foley et al. (2009) note the spectroscopic similarity between SN 2005hk and SN 2008ha at +15 and +8 days (after optical maximum), respectively, as well as their similarity with the subluminous Type IIP SN 2005cs at +44 days, while it was still on the photometric plateau 
(Pastorello et al. 2009). At such times Type IIP SNe have generally low polarizations (Leonard et al. 2006), seemingly similar to that observed by us for SN 2005hk. From a spectropolarimetric perspective, however, comparison of SN 2005hk with SN 2005cs while on the plateau is inappropriate. During the plateau phase, in Type IIP SNe, the photosphere is located in the hydrogen envelope. Due to the low ionization energy of $\mathrm{H}$, the shape of the photosphere is approximately spherical. Once the photosphere has descended below the $\mathrm{H}$ envelope, into the He layer (He being harder to ionize and excite), the shape of the photosphere more closely follows the shape of the underlying exciting material. Despite an asymmetric explosion, Leonard et al. (2006) observed that during the plateau SN 2004dj had a low polarization, which increased significantly once the plateau phase had ended. Given the H-deficient nature of SN 2002cxlike $\mathrm{SNe}$, a more appropriate comparison is with the H-deficient CCSNe, which have been observed (as listed in Table 4) to have significant continuum and line polarization for different species indicating the role of highly asymmetric explosion mechanisms. Highly asymmetric interiors have also been observed for the Type IIP SNe 2001dh and 2003gd (J. R. Maund et al. 2010, in preparation), which suggest that an asymmetric explosion mechanism is common to all these CCSN events.

It is interesting to note another ongoing controversy involving SNe Ia and SNe Ic. SN 2002ic was revealed by Hamuy et al. (2003) to resemble an SN Ia embedded in a hydrogen-rich medium. Hamuy et al. interpreted this as an SN Ia exploding with an evolved binary companion. Wang et al. (2004) showed that the hydrogen component was strongly polarized and argued that the geometry was consistent with an extended, clumpy, disklike structure such as observed in proto-planetary nebula stars. Hamuy et al. and Wang et al. (2004) identified other events of this category that were originally identified as Type IIn events, but which, on closer inspection and comparison with SN 2002ic, revealed an underlying Type I spectrum. More recently, Benetti et al. (2006) have challenged this interpretation, arguing that the underlying supernova in SN 2002ic more closely matched an SN Ic than an SN Ia.

In summary, the nature of the polarization of SN $2005 \mathrm{hk}$ is inconsistent with the polarization measured for CCSNe, in particular those of Type Ibc which have been generally observed to have very large polarization (especially line polarization) that increases with time.

\section{CONCLUSIONS}

SN 2005hk has been observed at two epochs, corresponding to maximum light and two weeks after maximum. After subtraction of the ISP component, the SN is observed to have a very low to null intrinsic polarization. A modulation in the Stokes parameters is observed across the Si II $\lambda 6355$ line being the most significant feature, at the first epoch, with $\Delta P=0.54 \% \pm 0.12 \%$.

At both epochs, a dominant axis can be fit to the data, although the data are poorly described by simple straight line fits on the Stokes $Q-U$ plane. While there is modulation in the dominant polarization component, at the first epoch, that is associated with line features in the flux spectrum, the absence of similar modulations in the orthogonal polarization component implies that the line-forming regions of the species in the flux spectrum share the same axial symmetry as that of the photosphere. The data at both epochs are classified as being of spectropolarimetric Type D1.

In comparison with previously published high $\mathrm{S} / \mathrm{N}$ observations of SN 2005hk at -4 days (Chornock et al. 2006), we observe a possible rotation of the polarization angle of the dominant axis, but it is unclear (due to the differences in $S / N$ ) if this represents a real rotation of the principal axis of symmetry with depth into the ejecta.

The low level of continuum polarization limits the asymmetry of the photosphere, at both epochs, to $<10 \%$. The low line polarization is suggestive that the line-forming regions are mixed, that different species occupy similar locations within the ejecta, and that they have a similar geometry to that of the photosphere. This is similar to the general behavior of Type Ia SNe and different from the chemically segregated ejecta observed for CCSNe.

We conclude that the spectropolarimetry of SN 2005hk is not inconsistent with some of the expectations of the deflagration explosion model. We find that in order to preserve the approximate spherical symmetry of the photosphere and the line-forming regions, any plume-like structures would be required to have a scale, in the plane of the sky, of $\leqslant 0.2 R_{\text {ph }}$ at both epochs. This would give the overall appearance of homogeneously mixed ejecta containing both the products of nucleosynthesis $(\mathrm{Fe}, \mathrm{Ni}$, $\mathrm{Co}$ ) and unburned $\mathrm{O}$ at all depths.

Spectral similarities between SN 2002cx-like events and lowluminosity Type IIP SNe are likely to be merely superficial. The differences in the observed spectropolarimetry of SN 2005hk with CCSNe suggests, from a geometry perspective, that these events have different underlying explosion mechanisms. Unfortunately, with spectropolarimetry of only one SN from the 2002cx-like class it is not possible to determine if these results are applicable to the entire class, in particular to the most peculiar member of this class, SN 2008ha.

The research of J.R.M. is funded through the Sophie \& Tycho Brahe Fellowship. The Dark Cosmology Centre is supported by the DNRF. The research of J.C.W. is supported in part by NSF grant AST-0707769. P.A.H is supported by the NSF grants AST 0708855 and 0703902 . The authors are grateful to the European Organization for Astronomical Research in the Southern Hemisphere for the generous allocation of observing time. They especially thank the staff of the Paranal Observatory for their competent and never-tiring support of this project in service mode. The authors thank the anonymous referee for useful comments and suggestions that have improved the paper.

\section{REFERENCES}

Benetti, S., Cappellaro, E., Turatto, M., Taubenberger, S., Harutyunyan, A., \& Valenti, S. 2006, ApJ, 653, L129

Bevington, P. R., \& Robinson, D. K. 2003, Data Reduction and Error Analysis for the Physical Sciences (Boston, MA: McGraw-Hill)

Branch, D., Baron, E., Thomas, R. C., Kasen, D., Li, W., \& Filippenko, A. V. 2004, PASP, 116, 903

Burket, J., \& Li, W. 2005, IAU Circ., 8625, 2

Cardelli, J. A., Clayton, G. C., \& Mathis, J. S. 1989, ApJ, 345, 245

Chandrasekhar, S. 1960, Radiative Transfer (New York: Dover)

Chornock, R., \& Filippenko, A. V. 2008, AJ, 136, 2227

Chornock, R., Filippenko, A. V., Branch, D., Foley, R. J., Jha, S., \& Li, W. 2006, PASP, 118, 722

Filippenko, A. V., et al. 1992, AJ, 104, 1543

Foley, R. J., et al. 2009, AJ, 138, 376

Gamezo, V. N., Khokhlov, A. M., \& Oran, E. S. 2004, Phys. Rev. Lett., 92, 211102

Hamuy, M., et al. 2003, Nature, 424, 651

Höflich, P. 1991, A\&A, 246, 481

Höflich, P., Gerardy, C. L., Marion, H., \& Quimby, R. 2006, New Astron. Rev., 50,470

Höflich, P., Mueller, E., \& Khokhlov, A. 1993, A\&A, 268, 570

Howell, D. A., Höflich, P., Wang, L., \& Wheeler, J. C. 2001, ApJ, 556, 302 
Jha, S., Branch, D., Chornock, R., Foley, R. J., Li, W., Swift, B. J., Casebeer, D., \& Filippenko, A. V. 2006, AJ, 132, 189

Kasen, D., Nugent, P., Thomas, R. C., \& Wang, L. 2004, ApJ, 610, 876

Kasen, D., et al. 2003, ApJ, 593, 788

Kawabata, K. S., et al. 2002, ApJ, 580, L39

Khokhlov, A. M. 1991, A\&A, 245, 114

Kozma, C., Fransson, C., Hillebrandt, W., Travaglio, C., Sollerman, J., Reinecke, M., Röpke, F. K., \& Spyromilio, J. 2005, A\&A, 437, 983

Leonard, D. C., Filippenko, A. V., Chornock, R., \& Foley, R. J. 2002, PASP, 114,1333

Leonard, D. C., Li, W., Filippenko, A. V., Foley, R. J., \& Chornock, R. 2005, ApJ, 632,450

Leonard, D. C., et al. 2006, Nature, 440, 505

Li, W., et al. 2003, PASP, 115, 453

Livne, E., Asida, S. M., \& Höflich, P. 2005, ApJ, 632, 443

Marietta, E., Burrows, A., \& Fryxell, B. 2000, ApJS, 128, 615

Maund, J. R. 2008, A\&A, 481, 913

Maund, J., Wheeler, J. C., Patat, F., Baade, D., Wang, L., \& Höflich, P. 2007a, MNRAS, 381, 201

Maund, J. R., Wheeler, J. C., Baade, D., Patat, F., Höflich, P., Wang, L., \& Clocchiatti, A. 2009, ApJ, 705, 1139

Maund, J., Wheeler, J. C., Patat, F., Wang, L., Baade, D., \& Höflich, P. 2007b, ApJ, 671, 1944

Mazzali, P. A., et al. 2005, ApJ, 623, L37

Munari, U., \& Zwitter, T. 1997, A\&A, 318, 269

Nomoto, K., Thielemann, F., \& Yokoi, K. 1984, ApJ, 286, 644

Pastorello, A., et al. 2009, MNRAS, 394, 2266

Patat, F., Baade, D., Höflich, P., Maund, J. R., Wang, L., \& Wheeler, J. C. 2009, A\&A, 508, 229

Patat, F., et al. 2007, Science, 317, 924
Patat, F., Maund, J. R., Benetti, S., Botticella, M. T., Cappellaro, E., Harutyunyan, A., \& Turatto, M. 2010, A\&A, 510, A108

Patat, F., \& Romaniello, M. 2006, PASP, 118, 146

Phillips, M. M., et al. 2007, PASP, 119, 360

Plewa, T., Calder, A. C., \& Lamb, D. Q. 2004, ApJL, 612, L37

Press, W. H., Teukolsky, S. A., Vetterling, W. T., \& Flannery, B. P. 1992, in Numerical Recipes in C. The Art of Scientific Computing, ed. W. H. Press, et al. (New York: Cambridge Univ. Press)

Röpke, F. K., Gieseler, M., Reinecke, M., Travaglio, C., \& Hillebrandt, W. 2006, A\&A, 453, 203

Sahu, D. K., et al. 2008, ApJ, 680, 580

Scarrott, S. M., Ward-Thompson, D., \& Warren-Smith, R. F. 1987, MNRAS 224, 299

Schlegel, D. J., Finkbeiner, D. P., \& Davis, M. 1998, ApJ, 500, 525

Serduke, F. J. D., Wong, D. S., \& Filippenko, A. V. 2005, CBE Tel., 269, 1

Serkowski, K., Mathewson, D. L., \& Ford, V. L. 1975, ApJ, 196, 261

Tanaka, M., Kawabata, K. S., Maeda, K., Hattori, T., \& Nomoto, K. 2008, ApJ, 689, 1191

Tanaka, M., et al. 2009, ApJ, 692, 1131

Tanaka, M., et al. 2010, ApJ, 714, 1209

Valenti, S., et al. 2009, Nature, 459, 674

Wang, L., Baade, D., Höflich, P., \& Wheeler, J. C. 2003a, ApJ, 592, 457

Wang, L., Baade, D., Höflich, P., Wheeler, J. C., Kawabata, K., Khokhlov, A., Nomoto, K., \& Patat, F. 2006, ApJ, 653, 490

Wang, L., Baade, D., Höflich, P., Wheeler, J. C., Kawabata, K., \& Nomoto, K. 2004, ApJ, 604, L53

Wang, L., Baade, D., \& Patat, F. 2007, Science, 315, 212

Wang, L., \& Wheeler, J. C. 2008, AR\&AA, 46, 433

Wang, L., Wheeler, J. C., \& Hoeflich, P. 1997, ApJL, 476, L27

Wang, L., et al. 2003b, ApJ, 591, 1110 